\title{
Correlation between Seismic Intensity Parameters of HHT-Based Synthetic Seismic Accelerograms and Damage Indices of Buildings
}

\author{
Eleni Vrochidou ${ }^{1}$, Petros Alvanitopoulos ${ }^{2}$, Ioannis Andreadis ${ }^{3}$ and Anaxagoras \\ Elenas $^{4}$ \\ 1,2,3 Department of Electrical and Computer Engineering, Democritus University of Thrace, \\ GR-67100 Xanthi, Greece \\ ${ }^{4}$ Department of Civil Engineering, Democritus University of Thrace, GR-67100 Xanthi, \\ Greece
}

\begin{abstract}
In this work, a correlation study between well-known seismic parameters and damage indices is presented. The correlation analysis is first performed on a set of natural seismic signals and afterwards on a set of artificial accelerograms generated from natural records. The artificial seismic signals are generated by a combination of techniques; the Hilbert-Huang transform and an optimization algorithm. In addition, they are compatible with the design spectra of a chosen seismic area. Results reveal that the seismic parameters of the synthetic earthquake accelerograms provide the same degree of correlation with the used damage indices as the natural earthquakes. Thus, the proposed synthetic accelerograms technique appropriately represents a seismic event and, therefore, it is a useful tool in earthquake engineering.
\end{abstract}

Keywords: Artificial seismic signals, Hilbert-Huang transform, correlation, seismic parameters, damage indices.

\section{Introduction}

In order to examine the resistance of a building, a dynamic analysis is required. Disaster scenarios need seismic accelerograms that could lead the building to the limits of its strength. Accelerograms are signal records of the acceleration versus time measured during an earthquake ground motion. Unfortunately, there is lack of natural strong motion earthquake records for some areas. Thus, there is need of artificial seismic records compatible with the design spectra of the area under observation. Methods for generating artificial earthquakes have been reported in the literature [14]. The presented methodology uses the Hilbert-Huang transform (HHT) which appears to be an effective technique for analyzing non-stationary and non-linear signals such as seismic signals [5].

The HHT decomposes the initial signal into a number of intrinsic mode functions (IMFs) and presents the results to the energy-frequency-time field, designing the socalled Hilbert spectrum. The IMFs extraction is based on the local characteristics of 
the signals and not on some predetermined functions. This is the key feature characterizing the HHT providing a more physical meaning to the analysis of the examined signals [5]. Moreover, the finite number of the extracted IMFs, decreases the computational complexity of the algorithm. An additional advantage of the method is that the initial seismic signal used for the synthesis of the artificial signal, could be of any intensity and could belong to any region. This provides flexibility, since there can be selected signals with finite number of points and decrease even more the complexity of the algorithm.

The main challenge is to generate realistic simulated earthquake records with the characteristics of natural signals. The proposed methodology is applied to an eightstorey reinforced concrete frame structure. Both seismic parameters and damage indices have been calculated from natural and artificial signals. A comparison between them is made and correlation analysis demonstrates that the generated earthquake signals have the same behavior as the natural ones, and have approximately the same distribution of values among the seismic parameters and the damage indices. For example, the spectrum displacement and spectrum velocity are correlated with the global damage index of Park/Ang by 0.905 and 0.929 , respectively for the natural accelerograms, while for the artificial accelerograms the values are 0.905 and 0.905 , respectively. Additionally, the correlation study reveals that the seismic parameters that are known to be most correlated with the damage indices [6] have the same degree of correlation for both natural and artificial signals.

\section{Earthquake Engineering}

The intensity of a seismic accelerogram is expressed with the help of several seismic parameters that are connected to the damages caused to a specific structure [6]. Damages are described by the damage indices (DIs). The maximum inter-storey drift ratio (MISDR) and the global damage index of Park/Ang ( $\left.\mathrm{DI}_{\mathrm{P} / \mathrm{A}}\right)$ are used to characterize the structural damage caused to buildings.

\subsection{Damage Indices}

The level of the post-seismic corruption of a structure can be evaluated by the MISDR [7] and is given by the following equation:

$$
\operatorname{MISDR}=\frac{|u|_{\max }}{h} 100[\%]
$$

where $|u|_{\max }$ is the maximum absolute inter-storey drift and $h$ the inter-storey height. In addition, the global DI after Park/Ang is also used as a damage index [7]: 


$$
D I_{P / A}=\frac{\sum_{i=0}^{n} D I_{L} E_{i}}{\sum_{i=0}^{n} E_{i}}
$$

where $E_{i}$ is the energy at the location $i, n$ is the number of location where the energy is estimated and $D I_{L}$ is the local DI given by the equation:

$$
D I_{L}=\frac{\theta_{m}-\theta_{r}}{\theta_{u}-\theta_{r}}+\frac{\beta}{M_{y} \theta_{u}} E_{T}
$$

where $\theta_{m}$ is the maximum rotation during the load history, $\theta_{r}$ is the recoverable rotation at unloading, $\theta_{u}$ is the ultimate rotation capacity, $\beta$ is a strength degrading parameter (0.1-0.15), $M_{y}$ is the yield moment of the section and $E_{T}$ is the dissipated hysteric energy.

\subsection{Seismic Parameters}

Accelerograms can be described by some well-known intensity parameters. In this work a set of 8 parameters has been utilized and is presented in Table 1 along with their literature references. The selection is based on the correlation degree of the parameters with the damage indices [6, 7].

Table 1. Seismic intensity parameters strong correlated with the structural damage indices.

\begin{tabular}{lll}
\hline No. & Seismic Intensity Parameters & Ref. \\
\hline 1 & Spectrum Intensity after Housner $\left(\mathrm{SI}_{\mathrm{H}}\right)$ & {$[8,9]$} \\
2 & Spectrum Intensity after Kappos $\left(\mathrm{SI}_{\mathrm{K}}\right)$ & {$[8,9]$} \\
3 & Spectral Displacement (SD) & {$[8,9]$} \\
4 & Spectral Velocity (SV) & {$[8,9]$} \\
5 & Spectral Acceleration (SA) & {$[8,9]$} \\
6 & Seismic Intensity after Fajfar/Vidic/Fischinger $\left(\mathrm{I}_{\mathrm{FVF}}\right)$ & {$[8,9]$} \\
7 & Peak Ground Velocity (PGV) & {$[8,9]$} \\
8 & Seismic Energy Input (E & {$[8]$} \\
\hline
\end{tabular}

\subsection{Concrete Frame Structure}

The examined reinforced concrete structure is depicted in Figure 1. The eigenfrequency of the 8-storey building is $0.85 \mathrm{~Hz}$ and its design is based on the recent Eurocode rules EC2 and EC8. The cross-sections of the beams are T-beams with $40 \mathrm{~cm}$ width, $20 \mathrm{~cm}$ slab thickness, $60 \mathrm{~cm}$ total beam height and $1.45 \mathrm{~m}$ effective slab width. The distance between the frames of the structure is $6 \mathrm{~m}$. The structure has 
been characterized as an "importance class II and ductility class medium"- structure according to the EC8 Eurocode. The subsoil is of type $\mathrm{C}$ and the region seismicity of category 2. External loads are taken under consideration and are incorporated into load combinations due to the rules of EC2 and EC8.

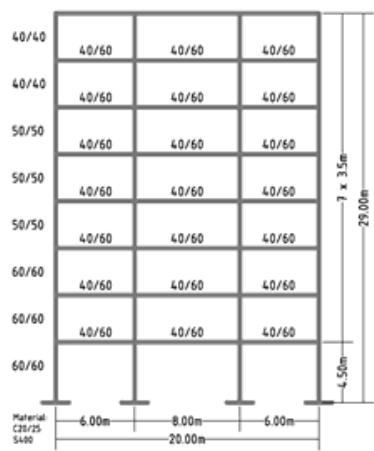

Fig. 1. Reinforced concrete frame structure.

\section{Synthesis of Artificial Accelerograms}

The proposed synthesis of artificial seismic signals is based on the HHT. According to HHT, each natural earthquake record can be decomposed into finite frequency and amplitude components. These components are appropriately modified to compose the artificial signal. This is achieved through an optimization algorithm which is used to minimize the mean square error between the response spectrum of the synthetic earthquake and the design spectrum of the selected seismic area.

\subsection{The Hilbert-Huang Transform}

The HHT [5] includes the Empirical Mode Decomposition (EMD) and the Hilbert Spectral Analysis (HSA).

The EMD decomposed the signal into a finite number of IMFs. The decomposition is based upon the local characteristics of the signal, thus it is an appropriate method for non-linear and non-stationary data analysis. Two conditions must be fulfilled for the generated IMFs:

1. the number of extrema and zero-crossings must be equal or differ the most by one and

2. at any point, the mean value of the envelop that is defined by the local maxima and minima must be equal to zero.

For a seismic signal $X(t)$, all the local extrema must be connected by a cubic spline so as to form the upper envelope of the signal $y_{\max }(t)$ and the same process is 
followed for the lower envelope $y_{\min }(t)$. The signal must be enclosed between these two envelopes. The mean value of the envelopes is:

$$
m_{1}(t)=\frac{\left(y_{\max }(t)-y_{\min }(t)\right)}{2}
$$

and the difference between the signal and the $m_{1}(t)$ is the first component $h_{1}$ :

$$
h_{1}(t)=X(t)-m_{1}(t)
$$

The procedure continues and now $h_{1}(t)$ is considered to be the signal, so:

$$
h_{11}(t)=h_{1}(t)-m_{11}(t)
$$

where $m_{11}(t)$ is the new mean of the two envelopes of $h_{1}(t)$. The same process is repeated for $k$ times and finally the $h_{1 k}(t)$ is:

$$
h_{1 k}(t)=h_{1(k-1)}(t)-m_{1 k}(t)
$$

where the $h_{1 k}(t)=c_{1}(t)$ is the first IMF. The process finishes when the standard deviation $S D \leq 0.2$ :

$$
S D=\sum_{t=0}^{T} \frac{\left[h_{1(k-1)}(t)-h_{1 k}(t)\right]^{2}}{h_{1(k-1)}^{2}(t)}
$$

where $T$ is the duration of the seismic signal. The residue $r_{1}(t)$ is derived by subtracting the first IMF from the initial signal:

$$
r_{1}(t)=X(t)-c_{1}(t)
$$

and it is considered as the new data. The new data is subjected to the same process until all $r_{j}(t)$ functions are obtained:

$$
r_{j}(t)=r_{j-1}(t)-c_{j}(t), j=2,3, \ldots, n .
$$

The iteration process stops when one of the two criteria is fulfilled:

1. either the residue $r_{n}(t)$ or the component $c_{n}(t)$ is less than a predetermined value or

2. the residue $r_{n}(t)$ is a monotonic function and no other IMF can be derived.

Finally, the initial signal is the sum of all IMFs plus the residue:

$$
X(t)=\sum_{j=1}^{n} c_{j}(t)+r_{n}(t)
$$

The Hilbert Spectral Analysis derives the instantaneous frequency data and forms the energy-frequency-time distribution by applying the HHT to each IMF. For all components $c_{j}(t)$, the Hilbert transform is: 


$$
y_{j}(t)=\frac{1}{\pi} \mathrm{P} \int_{-\infty}^{+\infty} \frac{c_{j}(\tau)}{t-\tau} d \tau
$$

where $\mathrm{P}$ is the Cauchy Principal Value. The combination of the IMF $c_{j}(t)$ and its Hilbert transform $y_{j}(t)$ form an analytic signal $z_{j}(t)$ :

$$
z_{j}(t)=c_{j}(t)+i y_{j}(t)=a_{j}(t) e^{i \theta_{j}(t)}
$$

where the amplitude $a_{j}(t)$ and the phase $\theta(t)$ are:

$$
\begin{gathered}
a_{j}(t)=\sqrt{c_{j}^{2}(t)+y_{j}^{2}(t)} \\
\theta_{j}(t)=\tan ^{-1} \frac{y_{j}(t)}{c_{j}(t)}
\end{gathered}
$$

The instantaneous frequency is given by the equation:

$$
\omega_{j}(t)=2 \pi f_{j}(t)=\frac{d \theta(t)}{d t}
$$

The IMF components are defined as follows:

$$
c_{j}(t)=\operatorname{Re}\left(a_{j}(t) e^{i \theta_{j}(t)}\right)=a_{j}(t) \cos \theta_{j}(t)
$$

where $\operatorname{Re}()$ is the real part. The initial signal can be written:

$$
X(t)=\operatorname{Re}\left(\sum_{j=1}^{n} a_{j}(t) \cos \left(\int 2 \pi f_{j}(t) d t\right)\right)
$$

In the previous equation, the residue is not invoked as it is a constant or a monotonic function. According to this equation both amplitude and frequency can be expressed as functions of time. The time-frequency distribution of the amplitude is known as the Hilbert spectrum $H(\omega, t)$.

\subsection{Method for Generating Artificial Seismic Accelerograms}

The objective of the proposed method is to supply earthquake engineering analysts with artificial seismic accelerograms compatible with the design spectrum of the current Greek antiseismic code [10] and it is a two step procedure.

The first step applies the HHT to the initial seismic signal which is decomposed into $n$ IMFs. The HHT amplitude and frequency components of the initial signal are then properly modified to form an artificial seismic signal $A E(x, t)$ which is given by the equation: 


$$
A E(x, t)=\operatorname{Re} \sum_{j=1}^{n}\left(x_{j} a_{j}(t)\right) e^{i x_{n+j} \int \omega_{j}(t) d t}
$$

where $x_{1}, x_{2}, \ldots, x_{n}$ are the amplitude scaling parameters and $x_{n+1}, x_{n+2}, \ldots, x_{2 n}$ are the frequency modifying parameters. The following equations describe the amplitude scaling and the frequency modification:

$$
\begin{gathered}
a_{i}(t)=x_{j} a_{i}(t), j=1,2, \ldots, n \\
\omega_{i}(t)=\omega_{i}(t)+x_{k}, k=n+1, \ldots, 2 n
\end{gathered}
$$

The second step solves the optimization problem so as the response spectrum of the synthetic signal to be compatible with the design spectrum of the area understudy. The optimization problem is actually finding the appropriate parameters $x=\left\{x_{1}, x_{2}, \ldots, x_{2 n}\right\}$ of the equations (20) and (21) to match the aforementioned spectrum. In particular, the genetic algorithm used, tries to minimize a fitness function which in our case is the Mean Square Error (MSE) between the two spectra:

$$
M S E=\frac{1}{n} \sum_{j=1}^{n}\left|S A_{\text {generated }}-S A_{t} \arg e t\right|^{2}
$$

where $n$ is the number of sample cases.

In this study, twenty natural seismic signals where used to generate synthetic spectrum-compatible seismic signals. The generated signals were afterwards submitted into a correlation study, to examine the relation between the seismic parameters and the damage indices, compared with the same correlation percentages of the initial seismic signals.

\section{Correlation Coefficients}

Correlation analysis proves the statistical relationship between two sets of data. There are many correlation coefficients; among them the most widely used are the Pearson correlation coefficient and the Spearman rank correlation coefficient.

The Pearson correlation shows how well the data fit a linear relationship, while the Spearman correlation shows how close the examined data are to monotone ranking. The latter coefficient is more important in the present study. For a set of $n$ measurements of $\mathrm{X}$ and $\mathrm{Y}$, where $i=1,2, \ldots, n$, the sample correlation coefficient is given by the equation:

$$
p_{\text {Spearman }}=1-\frac{6 \sum_{i=1}^{n} D^{2}}{n\left(n^{2}-1\right)}
$$

where $D$ is the difference between the ranking degree of $\mathrm{X}$ and $\mathrm{Y}$, respectively. 
In case the correlation coefficient has a value greater than 0.8 means that there is a strong connection between the parameters. On the other hand, values less than 0.45 demonstrate a weak connection between the parameters. All other cases between 0.45 and 0.8 reveal medium connection [6].

\section{Numerical Results}

In this study, twenty natural earthquake records were used as initial signals in order to generate synthetic accelerograms. Information about these seismic events is included in Table 2. Twenty artificial seismic accelerograms have been generated from these seismic signals. The objective was that the response acceleration spectrum of every generated signal to be compatible with the Greek design spectrum. Figure 2 displays the results obtained for the Friuli (Feltre station) earthquake record.

Table 2. Data for the 20 natural seismic signals.

\begin{tabular}{lll}
\hline Seismic Event & Station & Date \\
\hline Round Valley & Mcgee Creek Surface (USGS 1661) & $11 / 23 / 84$ \\
Coyote Lake & Coyote Lake Dam-San Martin, (CDMG 57217) & $08 / 06 / 79$ \\
Friuli & Conegliano & $05 / 06 / 76$ \\
Loma Prieta & Coyote Lake Dam Downst, (CDMG 57504) & $10 / 18 / 89$ \\
Coalinga & Chp, (CDMG 46T04) & $07 / 22 / 83$ \\
Alaska & Sitka Observatory & $07 / 30 / 72$ \\
Mt Lewis & Halls Valley, (CDMG 57191) & $03 / 31 / 86$ \\
San Fernando & Fort Tejon, (USGS 998) & $02 / 09 / 71$ \\
West Moreland & Brawley Airport, (USGS 5060) & $04 / 26 / 81$ \\
Oroville & Medical Center, (CIT 1544) & $08 / 02 / 75$ \\
Morgan Hill & Apeel 1E Hayward, (USGS/CDMG 1180) & $04 / 24 / 84$ \\
Dinar & Dinar & $01 / 10 / 95$ \\
Lytle Creek & Devils Canyon, (CDWR 620) & $09 / 12 / 70$ \\
Friuli & Barcis & $05 / 06 / 76$ \\
Borah Peak & Cem & $10 / 29 / 83$ \\
Morgan Hill & Capitola, (CDMG 47125) & $04 / 24 / 84$ \\
Mammoth Lakes & Fis & $05 / 27 / 80$ \\
Friuli & Feltre & $05 / 06 / 76$ \\
Kocaelli & Eregli, (ERD) & $08 / 17 / 99$ \\
Imperial Valley & Ec Co Center FF, (CDMG 5154) & $10 / 15 / 79$ \\
\hline
\end{tabular}

Figure 2(a) shows the original signal used to generate the synthetic earthquake signal that is shown in Figure 2(b). Figure 2(c) presents the response spectrum of the original signal, the response spectrum of the synthetic accelerogram and the Greek design spectrum of the considered seismic area. It is obvious from Figure 2(c) that the response spectrum of the generated signal is compatible with the desired design spectrum. This is also justified numerically, as the MSE for this experiment was $1.73 \times 10^{-3}$ which is significantly small. For this event, the response spectrum of the original accelerogram was lower than the design spectrum considered. 
Results are also promising for original signals that their response spectra are higher than the design spectrum. In this category belongs the seismic event of Dinar. The seismic parameters were calculated for all 20 original and artificial seismic accelerograms. Afterwards, the considered global damage indices were evaluated for each accelerogram, considered as seismic load on the examined frame structure. Finally, the Spearman rank correlation coefficient between the seismic parameters and the damage indices is calculated and presented in Table 3.
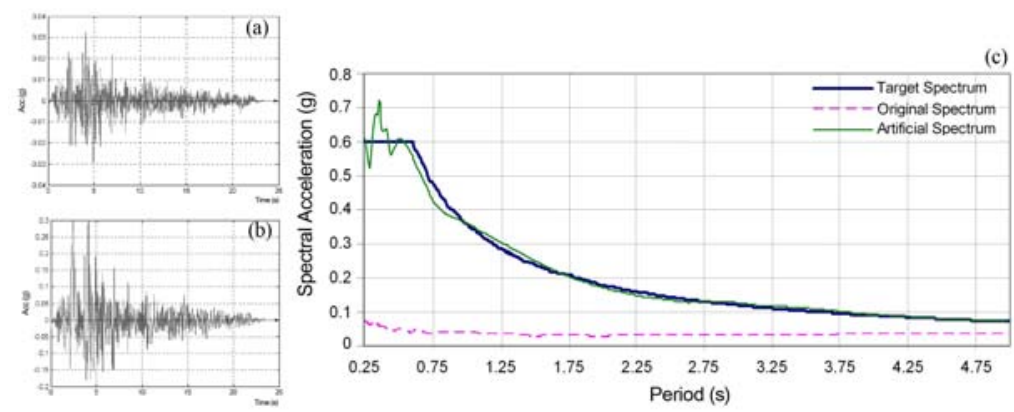

Fig. 2. (a) Friuli (Feltre station) seismic signal (b) synthetic generated signal and (c) response spectra and design spectrum.

As it can be seen from the numerical results of Table 3, the values of correlation are approximately the same for the natural acceleration records and artificial generated accelerograms. For example, the parameter SA for the natural signal is correlated with the MISDR and the $\mathrm{DI}_{\mathrm{P} / \mathrm{A}}$ by 0.833 and 0.881 , respectively. The same parameter for the artificial signals is correlated with the same damage indices by 0.762 and 0.833 respectively, which is almost the same degree as the natural records.

The degrees of interdependences for all 8 parameters are in the same range of values for natural and artificial signals. This indicates that the proposed method produces realistic simulations of earthquake signals.

Table 3. Spearman correlation coefficients between the seismic parameters and the damage indices.

\begin{tabular}{lllll}
\hline Seismic Intensity & Original Signal & \multicolumn{2}{c}{ Artificial Signal } \\
Parameters & MISDR & $\mathrm{DI}_{\mathrm{P} / \mathrm{A}}$ & MISDR & $\mathrm{DI}_{\mathrm{P} / \mathrm{A}}$ \\
\hline $\mathrm{SI}_{\mathrm{H}}$ & 0.810 & 0.786 & 0.929 & 0.857 \\
$\mathrm{SI}_{\mathrm{K}}$ & 0.786 & 0.762 & 0.810 & 0.881 \\
$\mathrm{SD}$ & 0.857 & 0.905 & 0.690 & 0.905 \\
$\mathrm{SV}$ & 0.857 & 0.929 & 0.690 & 0.905 \\
$\mathrm{SA}$ & 0.833 & 0.881 & 0.762 & 0.833 \\
$\mathrm{I}_{\mathrm{FVF}}$ & 0.857 & 0.929 & 0.690 & 0.905 \\
$\mathrm{PGV}$ & 0.905 & 0.952 & 0.690 & 0.905 \\
$\mathrm{E}_{\text {inp }}$ & 0.714 & 0.738 & 0.929 & 0.857 \\
\hline
\end{tabular}




\section{Conclusions}

The need of large sets of accelerograms for the dynamic analysis of structures and the shortage of natural records on many regions globally, justifies the search for generation of artificial spectrum compatible seismic signals. The proposed method generates spectrum compatible seismic signals based on the HHT and on a genetic algorithm that helps solve an optimization problem. One random seismic signal is used every time. This makes the method flexible as there can be chosen signals with a finite number of points and thus reduce the computational complexity of the method.

All generated signals were analyzed to prove that there are reliable simulations of natural signals. For this purpose, the values of the 8 well-known seismic parameters were calculated along with the damage indices for a certain frame structure, for both natural and artificial signals. The values extracted were then compared with the respective values of the initial seismic signals, and a correlation study was carried out. The numerical results indicate that the synthetic signals behave like real earthquake signals. The degree of interdependence was very similar for all damage indices, for natural and artificial earthquake signals, respectively. For example, the parameter SA is correlated with the $\mathrm{DI}_{\mathrm{P} / \mathrm{A}}$ for the natural and artificial earthquake signals by 0.881 and 0.833 , respectively.

\section{References}

1. Suarez, L. E. and Montejo, L. A.: Generation of artificial earthquakes via the wavelet transform. International Journal of Solids and Structures. 45, 5905--5919 (2005)

2. Mavroeidis, G. P. and Papageorgiou, A. S.: A Mathematical Representation of Near-Fault Ground Motions. Bulletin of the Seismological Society of America. 93, 1099--1131 (2003)

3. Das, S. and Gupta V. K.: Wavelet-based simulation of spectrum-compatible aftershock accelerograms. Earthquake Engineering \& Structural Dynamics. 37, 1333--1348 (2008)

4. Spanos, P.D., Giaralis, A. and Jie Li.: Synthesis of accelerograms compatible with the Chinese GB 50011-2001 design spectrum via harmonic wavelets: artificial and historic records. Earthquake Engineering and Engineering Vibration. 8, 189--206 (2009)

5. Huang, N. E., Shen, Z., Long, S. R. et al.: The empirical mode decomposition and the Hilbert spectrum for nonlinear and non-stationary time series analysis. Proceedings of the Royal Society A, 454, pp. 903--995, London (1998)

6. Elenas, A. and Meskouris, K.: Correlation study between seismic acceleration parameters and damage indices of structures. Engineering Structures. 23, 698--704 (2001)

7. Elenas, A: Correlation between seismic acceleration parameters and overall structural damage indices of buildings. Soil Dynamics and Earthquake Engineering 20(1-4), 93--100 (2000)

8. Alvanitopoulos, P., Andreadis I. and Elenas, A.: Interdependence Between Damage Indices and Ground Motion Parameters Based on Hilbert Huang Transform. Journal of Measurement Science \& Technology. 21, 1--14 (2010)

9. Alvanitopoulos, P., Papavasiliou, M., Andreadis I. and Elenas, A.: Seismic Feature Construction Based on the Hilbert Huang Transform. IEEE Transactions on Instrumentation \& Measurement. 61, 326--337 (2012)

10. EAK: National Greek Antiseismic Code. Earthquake Planning and Protection Organization (OASP) Publication, Athens (2003) 